\title{
STUDIES IN COLOR SENSITIVE PHOTOGRAPHIC PLATES AND METHODS OF SENSITIZING BY BATHING
}

\author{
By Francis M. Walters, jr., and Raymond Davis
}

\section{ABSTRACT}

The dyes which are used in color sensitizing ordinary (blue sensitive) plates by bathing require different methods for their most successful application. Pinaverdol, pinachrome, orthochrome $T$, and homocol may be used in water solutions, with or without ammonia, and are very little sensitive to the presence of electrolytes. Pinacyanol may be used in water solution, if the dry plates are first thoroughly washed, but gives greater sensitizing action with more fog and poorer keeping qualities when used with water, alcohol, and ammonia. Dicyanin gives comparatively little sensitizing except when used with water and alcohol and a fairly large per cent of ammonia. Films require the use of alcohol and ammonia to give the best results with pinacyanol.

Commercial panchromatic plates have their color sensitiveness improved by washing in water, without the increase in fog which occurs when they are treated with ammonia.

\section{CONTENTS}

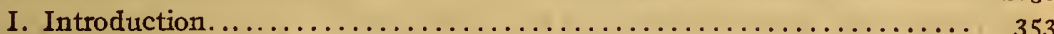

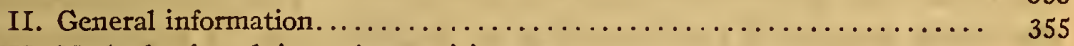

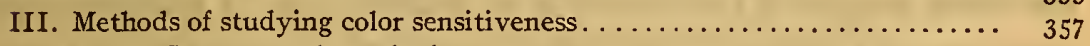

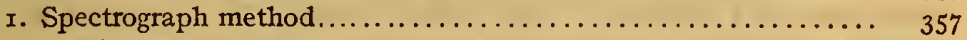

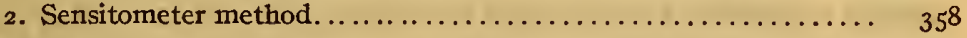

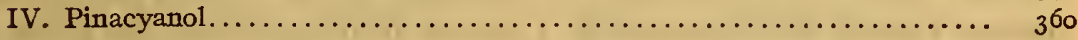

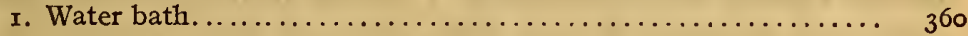

2. Water, alcohol, and ammonia bath................ $3^{61}$

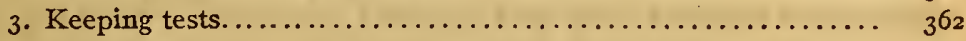

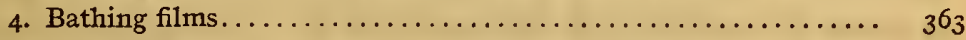

5. Speed of plates before and after bathing.............. ${ }_{3}^{6} 6_{5}$

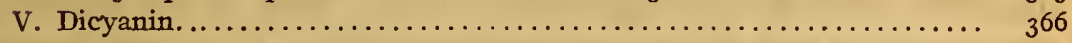

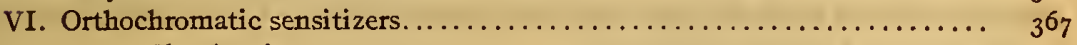

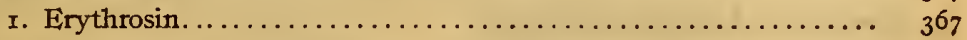

2. Pinaverdol, pinachrome, orthochrome $T$, and homocol ...... $3^{68}$

VII. Hypersensitizing commercial panchromatic plates. . . . . . . . . . $3^{68}$

VIII. Increase in speed by washing before exposure............... 369

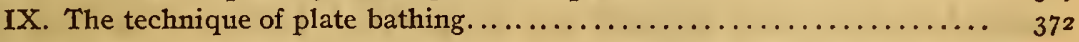

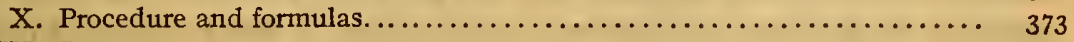

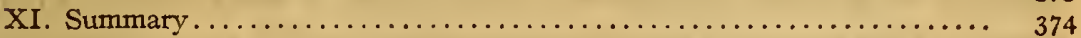

\section{INTRODUCTION}

Photographic plates and films owe their sensitiveness to light to the silver bromide embedded in the gelatine with which they are coated. Silver bromide as found in the ordinary plate (as 
well as silver chloride and silver iodide, which are also used in the preparation of photographic materials) are affected oniy by blue, violet, and ultra-violet light. Green, yellow, orange, and red have little or no effect, so that a photograph made on such a plate portrays blue, violet, and ultra-violet (which do not affect the eye) as white; while green (to which the eye is most sensitive), yellow, orange, and red are portrayed as black. This failure of the photographic process to render colors in black and white in the same order of luminosity as does the eye prevented for a long time the satisfactory photography of paintings, as well as the correct rendering of objects in nature.

By the addition of small amounts of certain dyes the photographic plate may, however, be made sensitive to green, yellow, orange, and red. Plates which are sensitive to the yellow-green as well as to the blue and violet are usually called orthochromatic, while plates which are sensitive also to the orange and red are called panchromatic or spectrum plates.

These three types of plates are illustrated in Fig. r. The letters at the botton of the spectrograms indicate the centers of the radiations usually recognized by the names: $V$, violet; $B$, blue; $\mathrm{G}$, green; $\mathrm{Y}$, yellow; $\mathrm{O}$, orange; $\mathrm{R}$, red. Beyond the blue and red are found the ultra-violet and the infra-red. The numbers express the wave length of the radiation in millimicrons (I millimicron= 0.000000 oor meter). The sensitiveness of the plate to any particular radiation is indicated by the height of the white part of the figure; thus a height equal to 4 spaces indicates that the plate is 4 times as sensitive to that radiation as it is to radiation where the white area reaches only to the third line and I6 times as sensitive as where it reaches only the second line. This sensitiveness refers, of course, only to the sensitiveness of the plate to the particular source used in making the spectrograms and not to its sensitiveness in terms of absolute energy.

Color-sensitive plates date from Vogel's discovery in $1873^{1}$. In one of his experiments a coliodion dry plate which had been stained yellow with aniline red to prevent halation showed, in addition to its blue and violet sensitiveness, a sensitiveness in the green. This region of sensitiveness corresponded to an absorption band in the spectrum of aniline red. He had much difficulty in the sensitizing of his plates, often obtaining only one satisfactory plate out of six attempts. With gelatine dry plates even greater

I Vogel, Handbuch der Photograpbie, 4th ed., I, p. 204; 1890. 

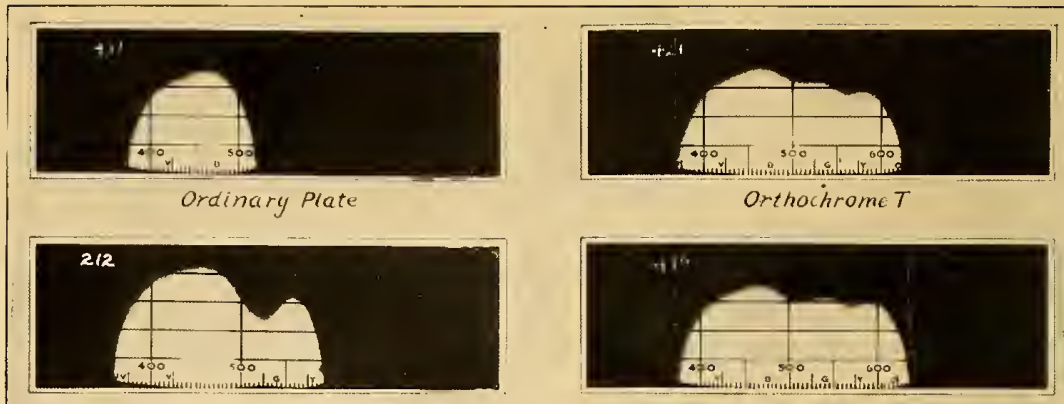

Orthochromatic Plate

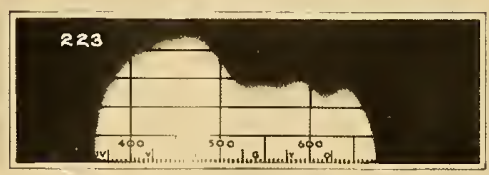

Panchromatic Plate

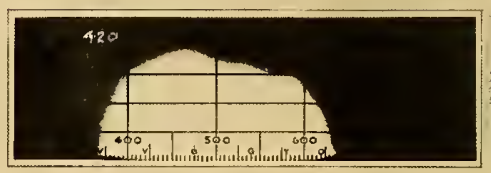

Pineverdol
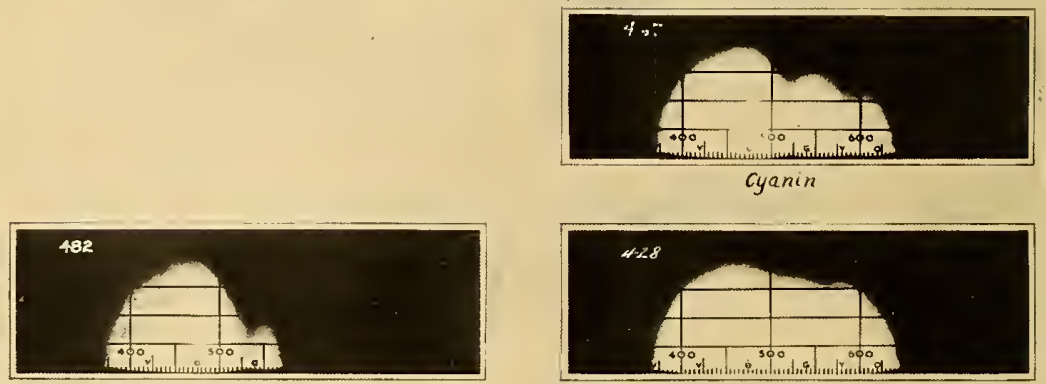

Eosin
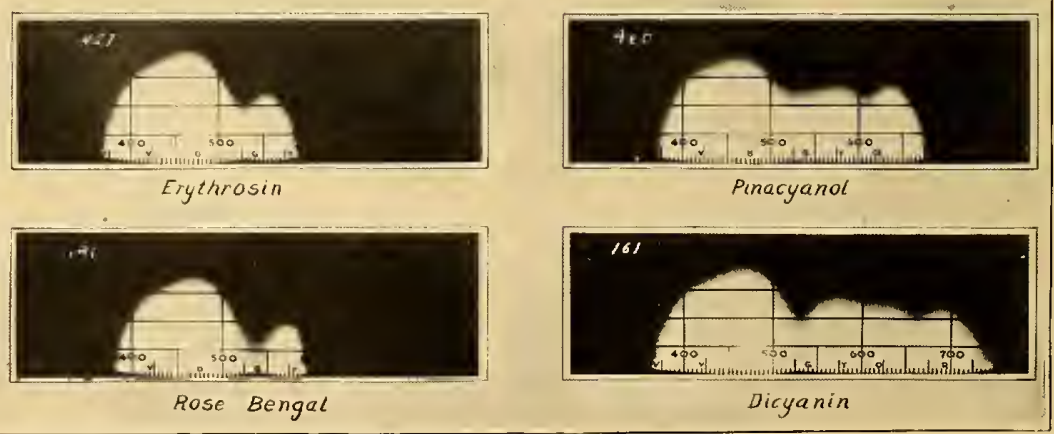

FIG. I.-Spectrograms of commercial and bathed plates

Spectrograms in the upper left-hand comer show the regions of color sensitativeness of the three types of commercial dry plates. The other spectrograms show the sensitiveness conferred to ordinary blue sensitive plates (Seed, $26 \mathrm{x}$ in this case) by bathing in solutions of sensitizing dyes. The name of the dye used is given under each spectrogram. The numbers indicate the wave length in $\mu \mu$. The letters $\mathrm{V}, \mathrm{B}, \mathrm{G}, \mathrm{Y}, \mathrm{O}$, and $\mathrm{R}$ indicate the colors-violet, blue, green, yellow, orange, and rcd. They are placcd at the center of the region recognized as being occupied by these colors. Beyond the violet and the red are the invisible rays, called ultraviolet and infra-red. All photographic plates are sensitive farthcr out into the ultra-violet than is indicated by these spectrograms. The cutting off of the ultra-violet is due to the glass used in the optical system of the spectrograph. A glass lens, such as is ordinarily used in a camera, has the same characteristic. 
difficulty was had until the notable discovery in 1882 of eosin ${ }^{2}$, which made silver bromide gelatine plates much more sensitive to yellow than any dye before used. Following this encouraging discovery of the sensitizing action of eosin many other dyes were investigated by Vogel, Schumann, Eder, and others, the number investigated by $\mathrm{Eder}^{3}$ and his students running into the hundreds. Comparatively few, however, found application in photography. The exceptions were cyanin, an orange sensitizer discovered by Vogel, and erythrosin, a strong yellow-green sensitizer discovered by Eder ${ }^{4}$ in 1884 . (See Fig. I.) Only one of these, erythrosin, remains in use to-day. Orthochromatic photography made little real progress until I 904-05, when König introduced pinachrome, orthochrome $T$, and pinacyanol (Fig. I). Pinacyanol sensitizes strongly in the red and serves as the foundation of commercial panchromatic plates.

\section{GENERAL INFORMATION}

Plates and films may be sensitized by two methods: (I) The dye may be incorporated in the emulsion at some stage in its preparation, usually immediately before coating, and (2) by bathing an ordinary blue sensitive dry plate in a solution of the dye. In general, the more sensitive plates result from bathing, but bathed plates often have the defect of not keeping.

The amount of dye required for sensitizing is very small. When incorporated in the emulsion, 2 to $4 \mathrm{mg}$ to $100 \mathrm{cc}$ of emulsion give the best results with most dyes, while in bathing the best concentration lies between I part in 25000 and I part in 75000 , although much smaller amounts give some sensitizing action.

In order to sensitize, a dye must combine with the silver bromide itself. Further, the dyestuffs which sensitize all fall into the class of so-called substantive dyes; that is, they dye substances directly. The center of the region which is sensitized by a given dye lies about 20 millimicrons farther toward the red than the center of the absorption band of the dye. ${ }^{5}$ Thus dyes which sensitize for the yellow-green are reddish in color, as, for example, erythrosin and pinaverdol. Dyes which sensitize for orange are purple, and red sensitizers are greenish. Eder found that the less silver iodide a silver bromide gelatin emulsion contained the better it

\footnotetext{
2 Attout Tailfer and Clayton: French patent No. 152645 of Dec. 13, I882, and Mar. 29, 1883.

3 Eder. Ausführliches Handbuch der Photographie, 2, p. 443; 1898.

- Eder and Valenta, Beiträge zur Photochemie und Spectralanalyse, Part III, p. 174. Wien: 1904.

- Eder, Handbuch, 3, p. 150.
} 
sensitized. Silver chloride sensitizes readily, but its original sensitiveness is so much less than that of silver bromide that the sensitized salt does not compare in rapidity with sensitized silver bromide. $^{6}$ An important characteristic of a dye suitable for sensitizing is its solubility. It must be soluble in water, or it must at least be capable of forming a fairly stable colloidal solution with it. Dyes soluble only in other solvents-for example, alcohol - can not penetrate the gelatine in such a manner as to dye the silver bromide. In addition, the dye should be of such a character as not to stain the gelatine so as to prevent the light from penetrating to the silver bromide. Were it not for this necessary characteristic, there are probably many dyes which would sensitize.

Before the war sensitizing dyes were principally imported from Germany. When this supply was cut off, the preparation of the dyes was undertaken by various laboratories in England and in the United States. The Ilford Co., of London, produced pinacyanol and pinaverdol, while in this country, the Eastman Kodak Co. are making orthochrome $\mathrm{T}$ as well. The color laboratory, Bureau of Chemistry, Department of Agriculture, has prepared pinaverdol, pinacyanol, and dicyanin. The success of these investigators has led to the preparation of entirely new sensitizers which promise marked improvement in panchromatic plates.

While commercial panchromatic plates are now sufficiently rapid for the photography of still objects and for three-color process work, there are certain problems which demand plates which are either more rapid or which are sensitive to radiations to which the panchromatic plate is not. Those who have these problems find it advisable to use ordinary plates sensitized by bathing. Important among the special problems are those in the field of spectroscopy, in which use is made of dicyanin to photograph infra-red spectra, and specially stained plates are indispensable in recording the visible spectrum of faint sources.

The directions which are furnished by the dye manufacturer for using the dye for bathing are at variance with the methods used at this Bureau and by some other workers. The work described in this paper was undertaken with the view of ascertaining the relation between the methods developed at this Bureau and the practice recommended by the dye makers.

- Eder, Handbuch, 3, p. 554 . 
Since pinacyanol is the most widely used red sensitizer and since good orthochromatic plates may be obtained commercially, the greater part of the investigation was done with this dye. Various methods and conditions of bathing were studied and keeping tests were made on plates sensitized by two methods of bathing. In addition to pinacyanol other important sensitizing dyes were studied.

\section{METHODS OF STUDYING COLOR SENSITIVENESS}

In studying the various ways of sensitizing by bathing two methods were employed: (I) The continuous spectrum of a source of white light was photographed on the plate, and (2) the

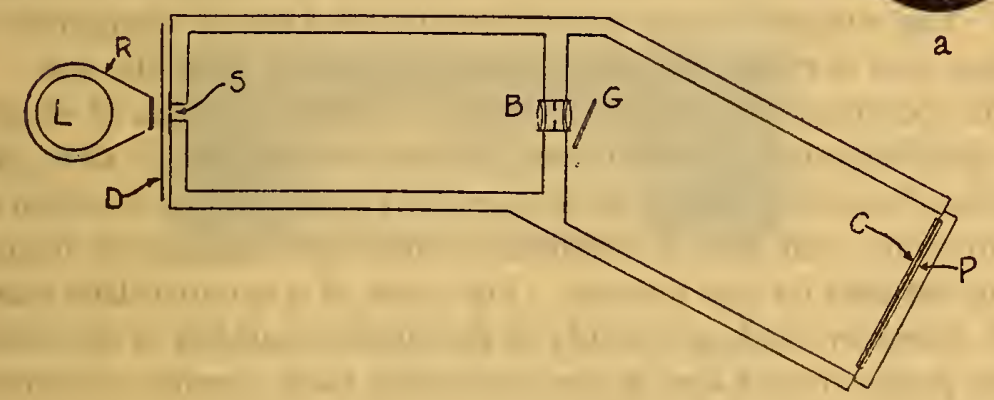

FiG. 2.-Diagram of specirograph

Disk for varying exposure alcng the slit located at $D$. $L$, lamp; $R$, refiector; $S$, slit; $B$, lens; $G$, replica grating; $c$, the wave-length scale which is in contact with photographic plate under test.

speed of the plate to white light was measured and the filter factors of the plate with a given set of color filters were determined.

\section{SPECTROGRAPH METHOD}

The spectrograph shown in Fig. 2 was designed for studying the color sensitiveness of plates.

The light source $(L)$ is a roo-watt tungsten lamp whose brightness is kept constant by adjusting a rheostat to maintain a constant current through the filament. Around the lamp is a white reflector $(R)$, while in front of the slit is a piece of ground glass, to give uniform illumination along the slit, which is 2 inches high. Between the ground glass and the slit is placed a rotating disk $(D)$, out of which is cut a variable aperture such that the exposure 


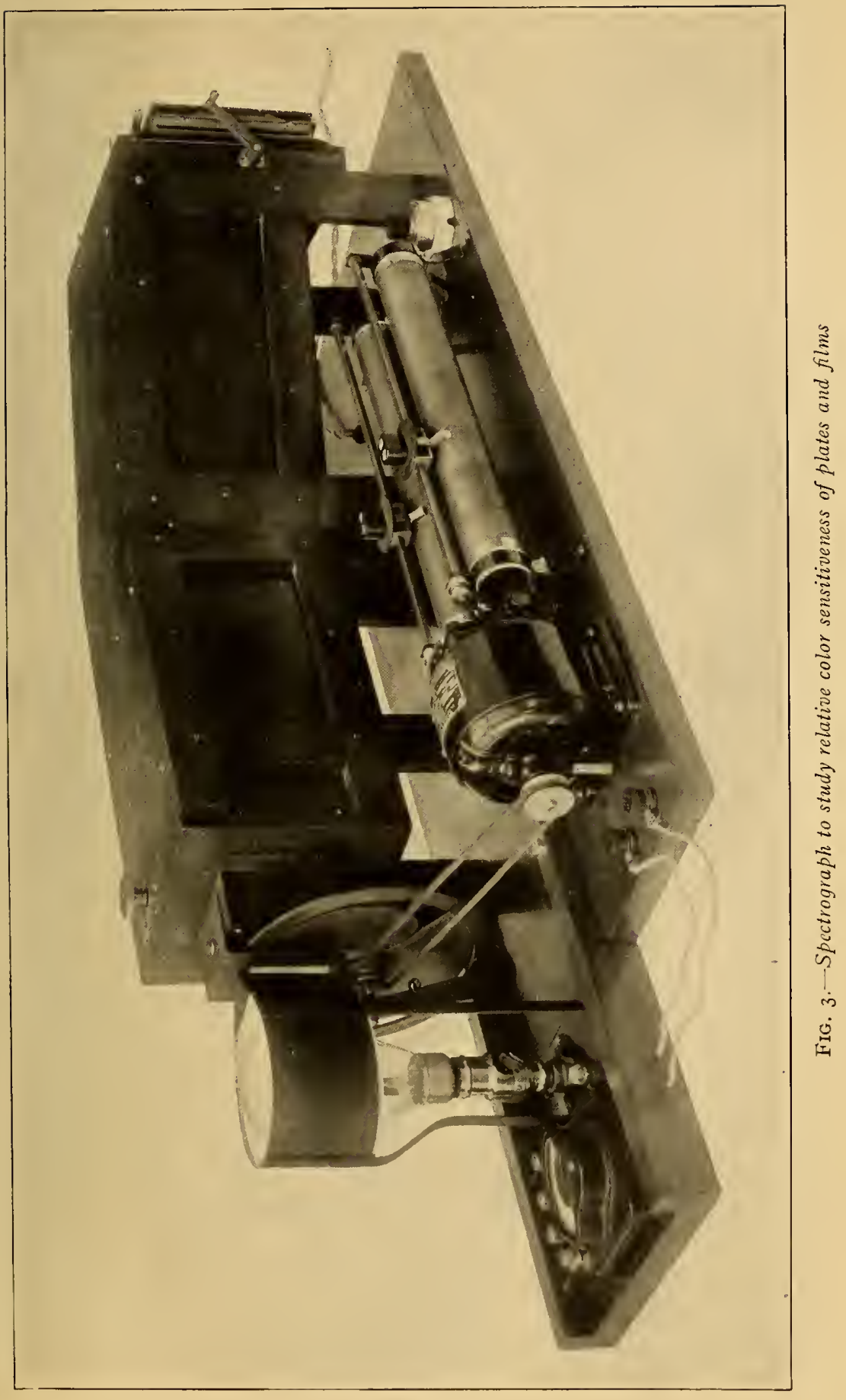


along the slit varies in a fixed manner with the distance from its ends. The series of horizontal lines on the spectrograms indicate geometrically decreasing exposures. The exposure at the first line from the bottom is one-fourth that at the bottom; at the second line, one-sixteenth; at the third, one sixty-fourth; at the fourth, one two-hundred-and-fifty-sixth; and at the top, one one-thousand-and-twenty-fourth. The light from the slit passes through first a photographic lens $(B)$, then a replica of a diffraction grating, which disperses it into a normal spectrum. Just in front of the plate $(P)$ is a screen $(C)$ bearing on it the reference lines which mark both intensities and wave lengths on the plate. The end of this screen, through which the red part of the spectrum passes, is stained with "rapid filter yellow" to screen out the second-order violet light which in the grating spectrum is superposed on the first order red.

As shown in Fig. 4, the tungsten lamp used for the investigation does not have the same spectral distribution of energy as does the sun. The tungsten lamp is deficient in the blue as compared to the sun and is relatively more intense in the red than the sun.

The spectrograph serves as a very convenient means of studying qualitatively the sensitizing action, showing as it does the regions of sensitiveness, both as to extent and as to the location of the maxima, and also, in addition to other detriments, the degree of fog induced by the process. For work of a quantitative character, however, such as a study of the time of bathing or the effect of the proportion of dye in the sensitizing bath, greater exactness is desirable. This is found in photographic sensitometry.

\section{SENSITOMETER METHOD}

Sensitometry consists in the exposure of a photographic material to a known source of light for definite intervals of time, the development of the material under standard conditions, and an interpretation of the blackening produced on the material in relation to the time of exposure and the intensity of the light acting. The details of the methods of sensitometry used in this work will be described in a forthcoming Bureau scientific paper.

Sensitometry is applied to the study of color sensitizing by using filters which transmit light of particular colors only. The filters used in this investigation, the relative spectrograms of which are shown in Fig. 5, are Wratten A, which gives the red sensitiveness; Wratten $G$, which gives very nearly the added sen- 
Scientific Papers of the Bureau of Standards, Vol. 17

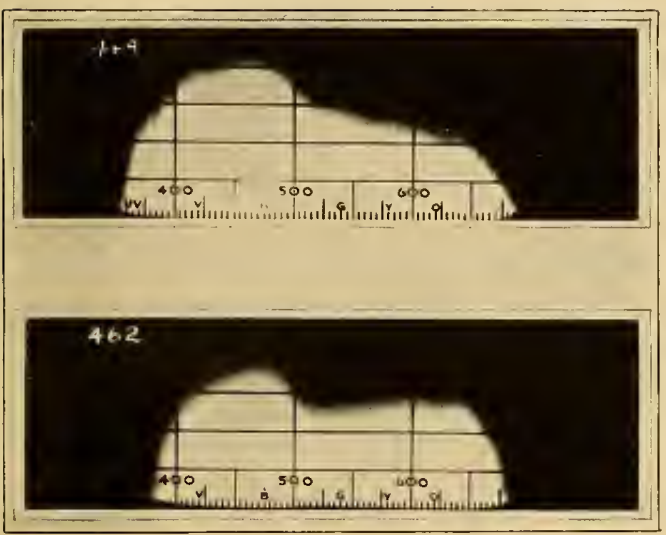

Sunlight

Tungsten lamp

FIG. 4.-Spectrograms showing the energy distribution of sunlight and of the tungsten lamp which was used in making the spcctrograms to show the sensitizing action of the various dyes

The vertical lines in the solar spectrum are the Fraunliofer lines. A comparison of the two spectrograms shows that the lamp has relatively more energy in the red than has the sun 
Scientific Papers of the Bureau of Standards, Vol. 17

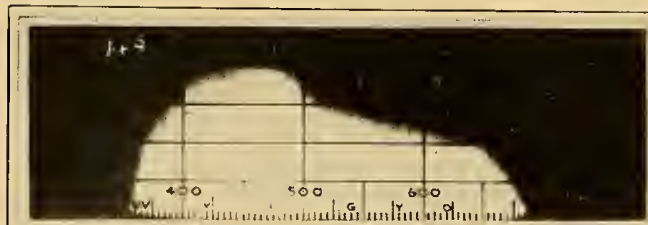

Without filter

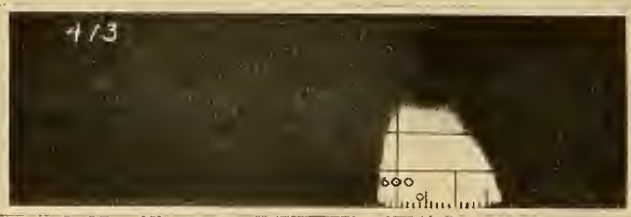

A filter

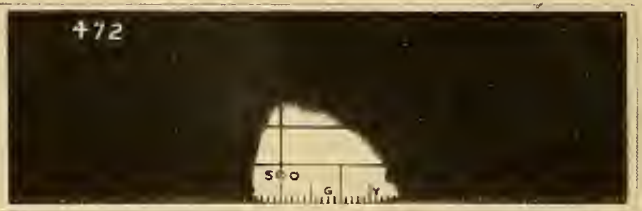

B filter

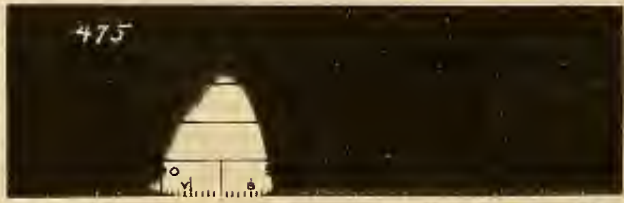

C filter

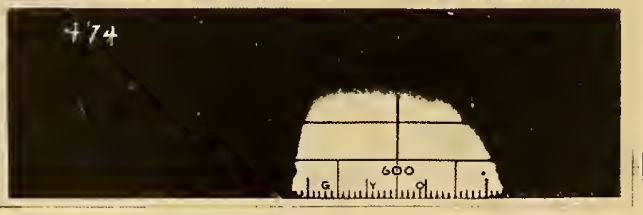

G filter

FIG. 5.-Spectrograms (with sunlight as the source) of a commercial panchromatic plate with the filters used in the speed tests

The $\mathbf{A}, \mathbf{B}$, and $\mathbf{C}$ filters select the red, green, and blue respectively, while the $\mathrm{G}$ transmits light of such color as to afford a measure of the added sensitiveness due to the dye 
sitiveness due to the dye; Wratten $B$, which gives the sensitiveness to green, and Wratten $\mathrm{C}$, to blue.

The speed or sensitiveness of the plate to the light transmitted by the filter may be measured by either introducing the filter between the light source and the plate in the exposing apparatus or by measuring the speed to white light and dividing this by the filter factor of the plate. When a number of filters are used, the second is the more rapid. The following procedure was, therefore, employed: Three strips from the sensitized plate were exposed in the sensitometer to a light source having approximately the spectral energy distribution of average noon sunlight, the strips were developed for different times, the densities measured and plotted against the logarithm of the time of exposure, and a speed determination made according to the method of Hurter and Driffield. ${ }^{7}$ When used with the four Wratten filters mentioned above, two strips from the plate served to determine the factors of the plate.

The filter factors were measured as described in Bureau Scientific Paper 409. The principle of this consists in varying the intensity of the light source until equal blackening is produced with equal exposure time with and without the filter. Since the filter factor gives the ratio of the total sensitiveness of the plate to its sensitiveness to the light transmitted by the filter, the filter factor and the speed of the plate without the filter give the data necessary to determine the speed of the plate with the filter. For example, if the speed of a plate to white light (average noon sunlight) is 400 and the filter factor with the A filter is Io the speed through the A filter is 40.

The method of stating color sensitiveness employed by Eder, ${ }^{8}$ Sheppard and Mees, ${ }^{9}$ and Wallace ${ }^{10}$ gives the added or chromatic sensitiveness in terms of the blue sensitiveness. This method we believe to be unsatisfactory, because the blue sensitiveness is decreased more or less by any process of sensitizing to other colors and because the ratio of the color sensitiveness to the blue sensitiveness does not give the best basis for comparison of one plate with another. The question to be answered is, Does this method or that give the greatest sensitiveness to a given color of light, regardless of what happens to the blue? This question

\footnotetext{
'Ferguson, "Photographic researches of Hurter and Driffeld," Royal Photographic Society, London ; 1920.

8 Éder and Valenta, Beitrage zur Photochemie II, p. 226.

- Sheppard and IIces; Investigations on the Theory of the Photograplic Process, p. 320, London; 1907.

${ }^{10}$ R. J. Wallace, Astrophys. Jour., 26, p. 305; 1907.
}

$62785^{\circ}-21-2$ 
is most directly answered by the speed of the plate to the light in question

\section{PINACYANOI}

Pinacyanol is, for many purposes, the most important sensitizer. Although it is commonly used in commercial panchromatic plates in conjunction with other sensitizers which give greater sensitiveness in the yellow-green, it alone gives a fairly good panchromatic plate.

\section{WATER BATH}

The following is typical of the directions given by the makers for the use of pinacyanol: Dissolve I part of pinacyanol in rooo parts of ethyl alcohol for the stock solution. To make red sensitive plates, bathe ordinary gelatine plates for two or three minutes in the following solutions: Water, 200 parts; stock solution, 3 parts. Wash well in running water, or frequent changes for several minutes, and dry in total darkness as quickly as possible in a current of warm, dry air free from dust.

If these directions are followed, it is found that the dye precipitates on standing after a plate has been bathed in it, or often before the plate has been sensitized, which results in the plate being spotted and lacking in sensitiveness. Pinacyanol is not soluble enough in cold water to wash away any dye deposited on the plate in the gelatine.

The addition of alum, ammonia, potassium bromide, and other electrolytes to the dye bath (water $200 \mathrm{cc}$, pinacyanol stock solution $3 \mathrm{cc}$ ) causes flocculation of the dye. This may indicate that the bath is not a true solution, but a colloidal solution or dispersoid.

This phenomenon suggested to the writers that the failure of the water bath in sensitizing ordinary commercial plates might be due to the presence of electrolytes in the emulsion. It is customary to add to the emulsion immediately before coating a small quantity of chrome alum to harden the gelatine, so that it will withstand alkaline development at room temperatures. There may also be present various other electrolytes, such as soluble bromides to restrain fog. ${ }^{11}$ If present, these soluble salts would come to the surface of the plate in drying and would thus be in a position to cause the speedy flocculation of the dye bath.

Accordingly, we tried washing the plates in tap water before bathing in a water solution of pinacyanol and found that the plates then did not cause the flocculation of the dye bath. The 
time for bathing is also decreased by this operation, since the gelatine swells somewhat and permits diffusion to take place more rapidly. With a washing time of five minutes the time required for sensitizing is decreased to two minutes.

While not so fast as plates sensitized in a bath containing ammonia, the water-bathed plates show considerably less initial fog and also keep much better.

Another point of importance is that if a plate be examined after removal from the dye bath there is found on it a considerable amount of the dye which is not removed by washing in water. In addition, there is a considerable staining of the gelatine, which decreases by its screening action the sensitiveness of the plate. Rinsing the plate in ethyl alcohol after the dye bath will remove to a large extent the dye dissolved in the gelatine, as well as that on the surface, while not affecting that which has combined with the silver bromide and to which the sensitizing action is due. That this is true may be shown by the fact that prolonged bathing in alcohol after sensitizing in the water bath does not decrease the color sensitiveness. It is common experience that bathed plates must be dried rapidly to secure speed and freedom from fog. ${ }^{12}$ In addition, therefore, to furnishing cleaner plates, the rinse in alcohol aids in drying the plates. The drying is accelerated by the alcohol, which replaces to a large extent the water in the gelatine film.

\section{WATER, ALCOHOL, AND AMMONIA BATH}

It has been observed (particularly with erythrosin) that the addition of ammonia to the dye bath increases the sensitizing action of the dye. If ammonia be added to a water bath of pinacyanol, flocculation occurs and prevents sensitizing. However, ammonia may be safely added to the dye bath if sufficient alcohol be present. Thus the formula used at this Bureau calls for-

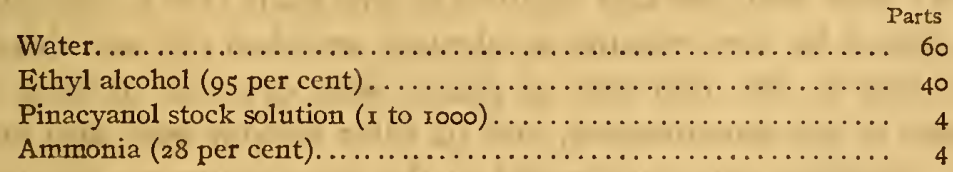

For ordinary work this quantity of ammonia usually gives too much fog and is recommended only in the case of plates which 
are used for position measurements, as in spectroscopy. If the amount of ammonia be cut from 4 to $2 \mathrm{cc}$, the sensitizing action is nearly as great and the fog is much less. Compared with plates bathed in water and pinacyanol, the sensitizing action is twice as great. As shown in Fig. 6, the sensitizing action of a bath composed of water and alcohol with no ammonia is less than the bath containing water only. For use in spectroscopy the ammonia method has the particular advantage of extending the region of sensitiveness, so that lines further in the red may be photographed. (See Fig. 7.)

It should be emphasized, however, that ammonia as a sensitizer reduces the keeping qualities of plates below those of other sensitizers.

\section{KEEPING TESTS}

In making the test on the relative keeping quality of plates sensitized by the two methods of bathing a slow portrait plate which works fairly free from fog was used. A dozen of these were bathed by each method on a clear, dry day.

The water bathing was carried out as follows: The plates were placed in the cage of a developing tank, washed in running tap water (temperature $18^{\circ} \mathrm{C}$ ) for five minutes, then transferred to a bath composed of tap water I $900 \mathrm{cc}$, pinacyanol stock solution (I to IO00) $38 \mathrm{cc}$. After bathing for two minutes they were placed in alcohol for two minutes, swabbed off, and dried in a light-tight cabinet, through which air at room temperature was forced by an electric fan. When dry the plates were packed face to face in an ordinary plate box.

The other dozen plates were placed directly in a tank containing a bath composed of-

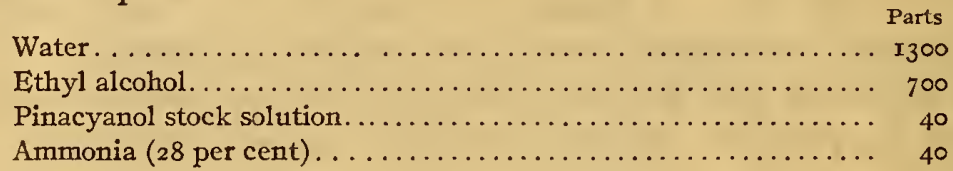

After four and one-half minutes in this bath $\left(18^{\circ} \mathrm{C}\right)$ the plates were rinsed for two minutes in alcohol, swabbed off, and dried.

As soon as the two sets of plates were dry one of each was exposed in the sensitometer, and its filter factors were also measured. Another pair was treated in the same manner a few hours later on the same day. Then tests were made once a day for a few days and finally at intervals of about a week. 
Scientific Papers of the Bureau of Standards, Vol. 17

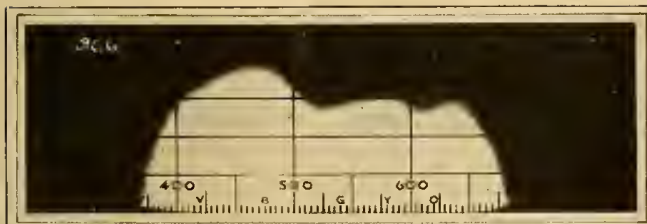

Pinacyanol and water; five minutes prewash in tap water

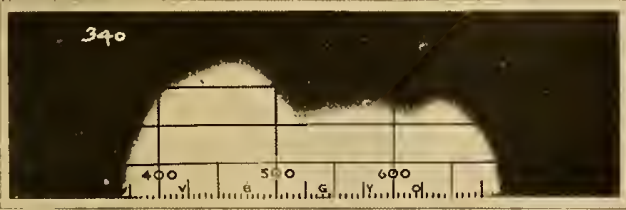

Pinacyanol, water, and alcohol

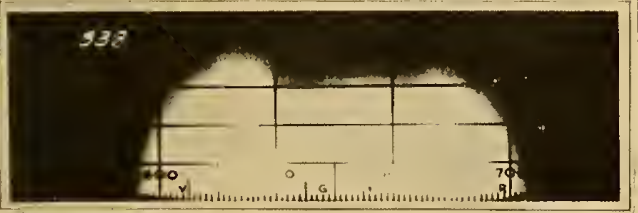

Pinacyanol, water, ammonia, and alcohol

FIG. 6.-Spectrograms showing the difference in the sensitizing action of pinacyanol when used in baths of various composition

Notice the incrcased action with ammonia, both in the height of the maxima and in the extension of the region of the sensitizing 
Scientific Papers of the Bureau of Standards, Vol. 17

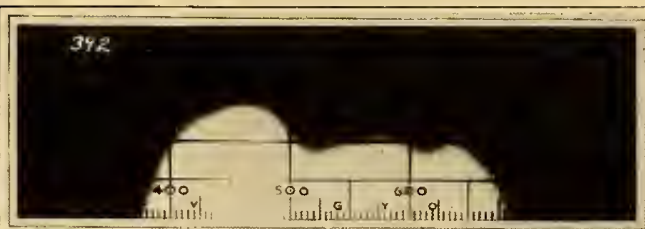

$\frac{1}{2} \mathrm{cc}$ ammonia

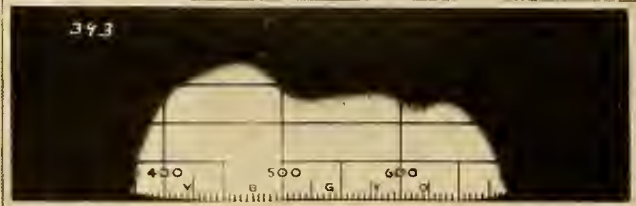

1 cc ammonia

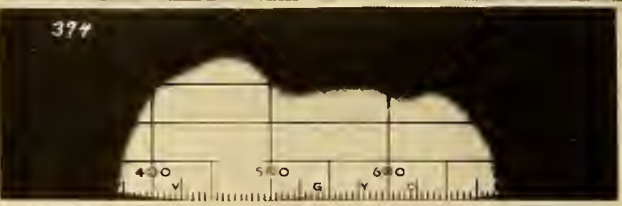

2 cc ammonia

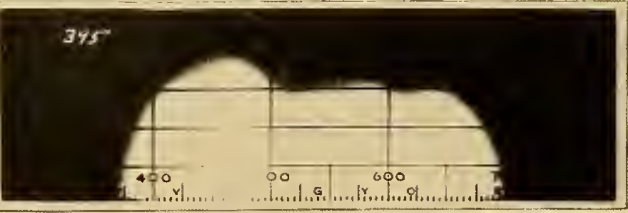

4 cc ammonia

Fig. 7.-The effect of various quantities of ammonia added to a dye bath of water and alcohol

Note that there is comparatively little difference between the $2 \mathrm{cc}$ and the $4 \mathrm{cc}$ in sensitizing action. However, the fog which is not indicated in the cuts increases with the proportion of ammcria 
The curves in Fig. 8 show the variation in speed ${ }^{13}$ to white light and the fog ${ }^{14}$ of the plates sensitized by the two methods.

Fig. 9 shows the characteristic curves for a plate sensitized with pinacyanol, water, alcohol, and ammonia when the plate was usable, while Fig. Io shows the characteristic curves of a similar plate after it had become unfit for use.

The variation in speed through filters with time of keeping is shown in Figs. II and I2. If these curves be compared with those for the variation in speed to white light (Fig. 8), it will be seen that while the speed to white light was decreasing the speed due

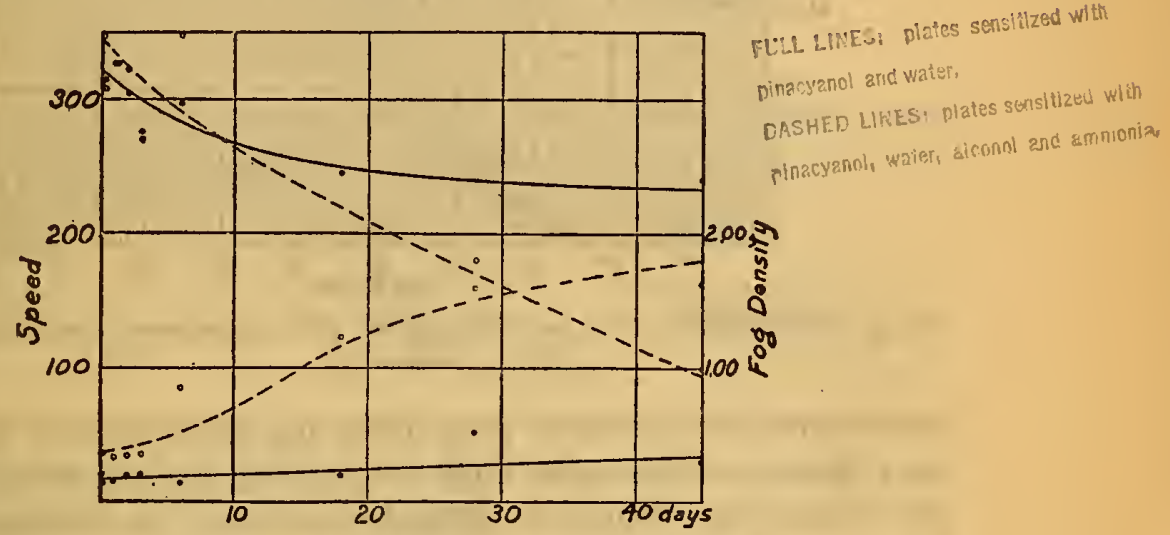

FIG. 8.-Keeping tests on plates sensitized with pinacyanol

The curves which start at the upper leit-hand corner of the graph show the speed to white light, while those that start at the lower left-hand corner show fog (for unit development to unit contrast) measured in density. Fiull lines=plates sensitized in pinacyanol, water, alcohol, and ammonia.

Nore.-The fog is given for $\gamma=\mathrm{x}$; that is, a development such that the contrast of the negative is the same as that of the subject.

to the added sensitiveness conferred by the pinacyanol did not decrease at quite the same rate.

\section{BATHING FIIMS}

In sensitizing films with pinacyanol it was found that the method of prewashing and bathing in a water solution of the dye

13 "Speed" as used here is measured by the method of Hurter and Driffeld. Briefly, this is as follows; When the density due to exposure to light is plotted against the logarithm of the time of exposure some of the points will lie on a straight line. If samples of the plate which have received a set of exposures are developed for different lengths of time, their respective straight line parts extended intersect nearly in a point on the exposure axis. The value of the exposure at this intersection point is called the "inertia" of the plate. The speed numbers used by us are ro divided by the inertia, so that the greater the speed number the faster the plate. This number was selected instead of Hurter and Driffield's $34 / \mathrm{I}$ because the light source employed by them (the sperm candle) is deficient in the blue as compared with our light source, which approximates average noon sunlight in its distribution of spectral energy. It should be noticed that for this reason our speed numbers are not convertible into Hurter and Driffield numbers.

${ }^{14} \mathrm{Fog}$ is measured as density: Density is defined as $\log { }^{10}(I / T)$, where $T$ (transmission) is the ratio of the transmitted to the incident light. Thus a fog density of 0.30 transmits one-half of the light falling on a plate; a density of 1.00 , one-tenth of the incident light and a density of 2 , one one-hundredth.

$62785^{\circ}-21-3$ 
was not successful and that to get adequate sensitizing action it was necessary to use the bath containing water, alcohol, and ammonia. Films have a tendency to give more fog with bathing than do some plates. It has been remarked by some that any

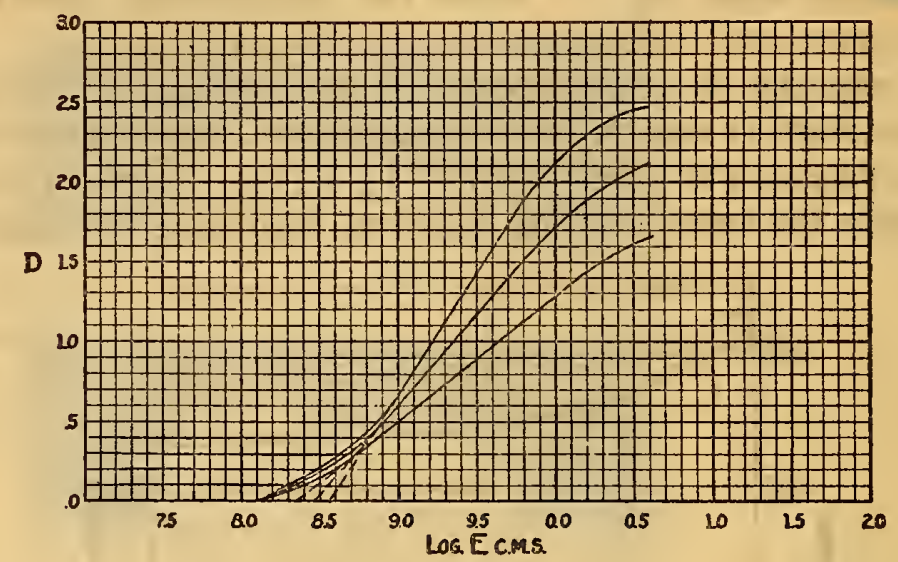

FIG. 9.-Characteristic curves for freshly, bathed plate (pinacyanol, water, alcohol, and ammonia)

orthochromatic emulsion gives more fog when bathed than ordinary plates, and suitable films for bathing are not obtainable except with some degree of orthochromatism. In bathing films it is necessary to exercise considerable care that nothing comes in

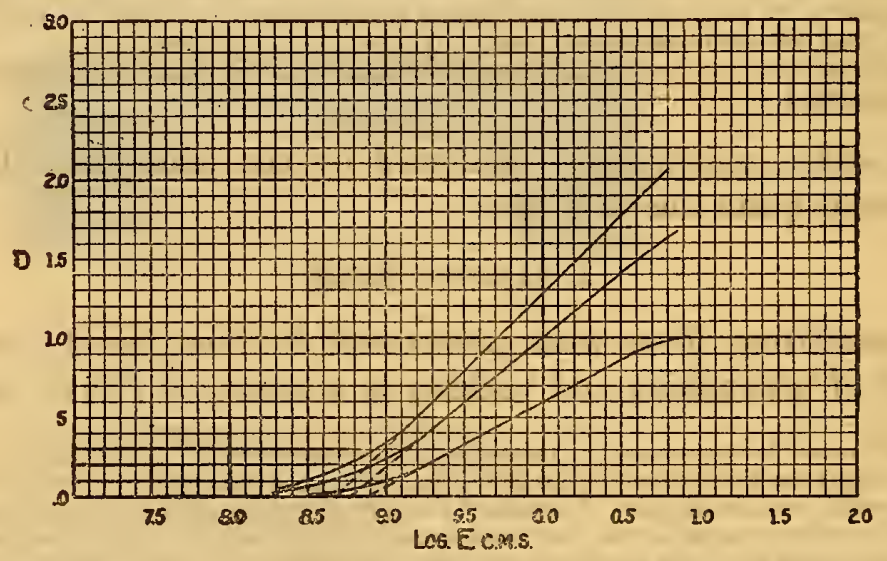

FIG. 10.-Characteristic curves for old bathed plaies (pinacyanol, water, alcohol, and ammonia)

contact with either side of the film, since the back is usually coated with gelatine to make the film noncurling. If the back of the film becomes scratched, these scratches will cause defects in the print. 


\section{SPEED OF PLATES BEFORE AND AFTER BATHING}

To study the comparative sensitizing action on plates having different characteristics as to speed and contrast, four plates made by the same manufacturer were selected, a fast plate, a mediumspeed plate, a slow plate, and a process plate. The plates were

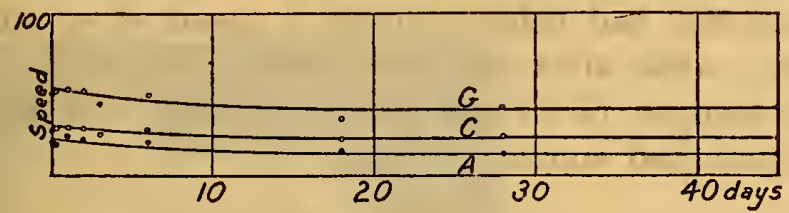

FIG. Ix.-Plates sensitized with pinacyanol and water

sensitized in a bath containing pinacyanol, water, alcohol, and ammonia. Table I shows the results obtained:

TABLE 1

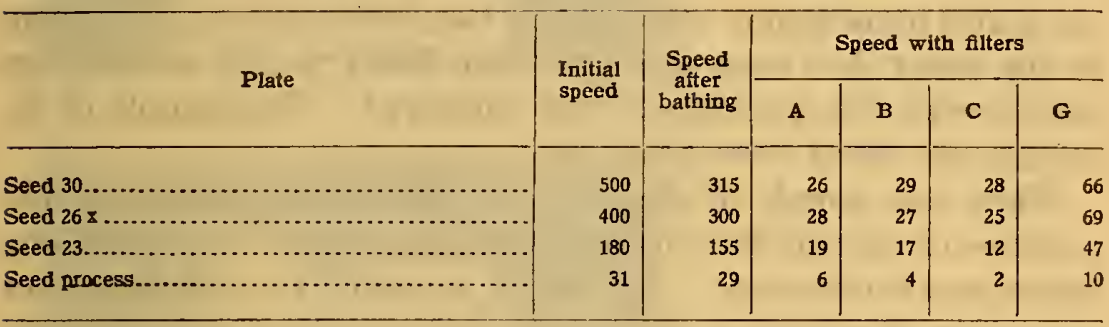

These results indicate that more added sensitiveness is conferred by the dye bath on slow plates than on fast ones. It may be concluded that when speed is not the factor of prime impor-

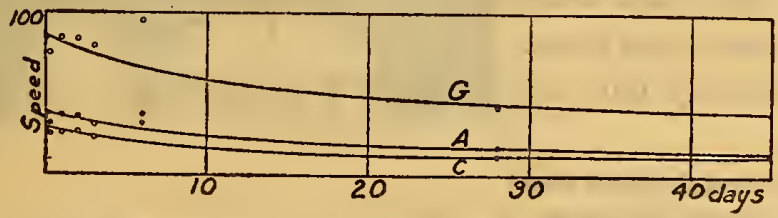

FIG. 12.-Plates sensitized with pinacyanol, water, alcohol, and ammonia

The $G$ filter gives very nearly the added sensitiveness due to the dye. The $A$ filter shows the red sensitiveness. The $C$ filter shows the blue sensitiveness. If these curves be compared with those in Fig. 8 , it will be observed that the color sensitiveness does not decrease as rapidly with time as the sensitiveness to white light.

tance, the plate chosen for bathing should be selected on the basis of its freedom from fog, its developing characteristics (contrast), and its resolving power.

The spectrograms of Fig. 13 show that the sensitiveness added by pinacyanol is about the same for the $30,26 \times$, and 23 , although 
the blue sensitiveness which the plates had originally still falls in the same order as the speed before bathing. Relatively the process plate has acquired a much greater sensitiveness than the other three plates, although its actual speed is far below them.

It may be remarked here that the blue sensitiveness of a plate is decreased by sensitizing. Thus the plates which were used for the keeping test had before bathing a speed of 55 with the $\mathrm{C}$ filter (blue), while after sensitizing with pinacyanol and water the speed dropped to 28 and after sensitizing with pinacyanol, water, alcohol, and ammonia to 26 .

\section{DICYANIN}

The makers' instructions for the use of dicyanin call for much the same procedure as in the case of pinacyanol. However, with dicyanin, even prewashing failed to give satisfactory sensitizing with the water bath. Mees ${ }^{15}$ states that it is necessary to bathe the plates immediately after adding the stock solution of dicyanin to the water, but even this procedure failed to give satisfactory results with the specimen of dye employed. This sample of dicyanin was about three years old.

When this sample of dicyanin was used with ammonia in the presence of enough alcohol to prevent flocculation, the sensitizing action was satisfactory. The use of ammonia has the important advantage of extending the sensitiveness to longer wave lengths, as well as increasing the action of the dye at its maxima. It is due to the use of ammonia that certain investigators ${ }^{16}$ have been able to photograph in the infra-red as far as Iooo $\mu$, while those who have not used ammonia with dicyanin have failed to photograph further than $800 \mu$.

A satisfactory bath for dicyanin is as follows:

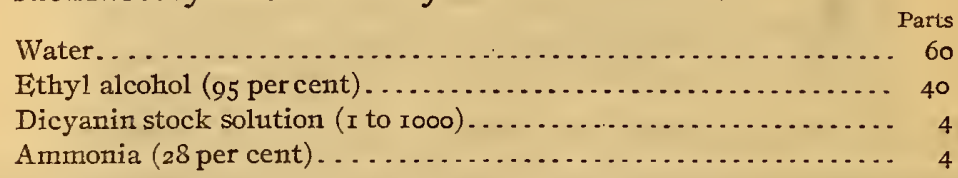

As shown in Fig. 14, the more ammonia used the greater the sensitizing action, but the proportion of ammonia is limited by the fog it induces.

Dicyanin requires more care in its use than the other photosensitizing dyes. The solid dicyanin should be bronze green in

${ }^{25}$ Mees and Wratten, Photographic Jour, 58, p. 25, 1908 (Newton and Bull).

16 B. S. Bull., 14, p. 376, 1917. 
Scienlific Papers of the Bureaul of Standards, Vol. 17

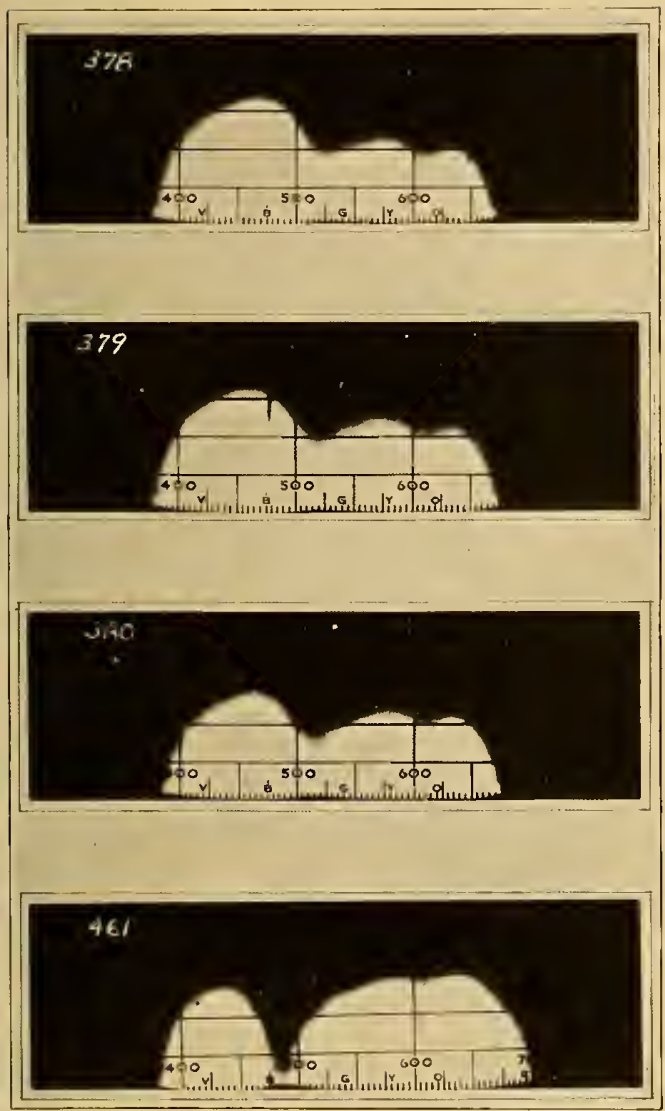

Seed 30

Seed $26 x$

Seed 23

Seed Process (60-minute exposure)

FIG. I3.-How effect of bathing with pinacyanol varies with the type of plate bathed

The plates shown represent the speed and contrast. It is to be observed that the faster the plate the less the addcd sensitiveness relatively 


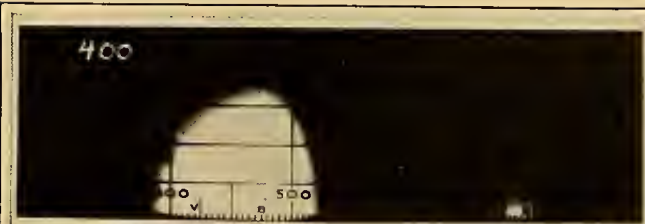

401

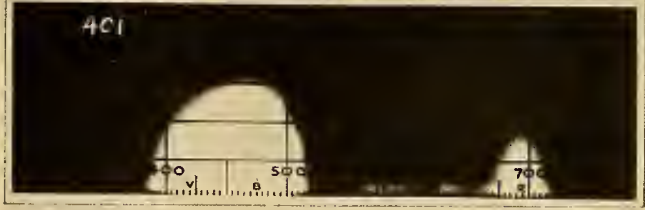

402

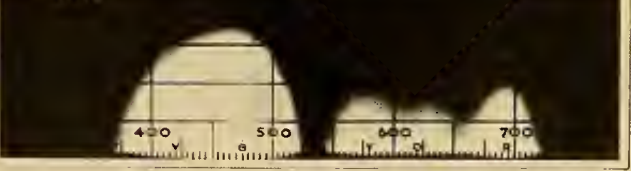

\section{3}
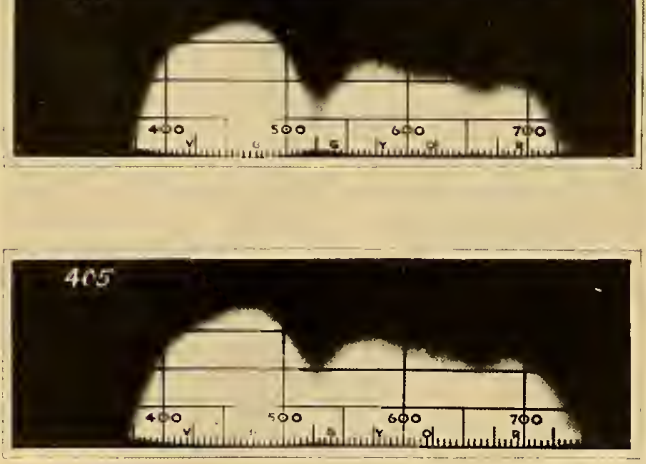

FiG. 14.-Spectrograms showing the difference in the sensitizing action of dicyanin when used in the baths of various composition

The use of sufficient ammonia in the bath not only increases the sensitizing action of dicyanin at its maxima, but extends its action into the infra-red as far as rouo $\mu \mu$
Dicyanin and water; 5 minutes prewash in tap water

Dicyanin, water, and alcohol

Dicyanin, water, alcohol, and $\frac{2}{2}$ per cent ammonia

Dicyanin, water, alcohol, and 1 per cent ammonia

Dicyanin, water, alcohol, and 4 per cent ammonia 
color. If it is not, particularly if it appears brownish, the dye has deteriorated and should not be used. The dye must be kept cool and dry in order to retain its sensitizing power. A freshly mixed bath of dicyanin is a bright blue-green color. As soon as the dye deteriorates the color changes considerably, the solution appears dull, and in addition shows a transmission band in the red. Such a solution should not be used. The temperature of the bath should not exceed $18^{\circ} \mathrm{C}$, if plates reasonably free from fog are to be obtained. The alcohol rinse after bathing is decidedly beneficial.

\section{ORTHOCHROMATIC SENSITIZERS}

The other important sensitizers investigated were pinaverdol, pinachrome, homocol, orthochrome $\mathrm{T}$, and erythrosin. These dyes are by no means as sensitive as pinacyanol and dicyanin to the action of electrolytes; they do not flocculate so readily in the presence of ammonia, potassium bromide, and alum. With the orthochromatic sensitizers the action of ammonia is most marked with erythrosin and homocol. The other dyes show a slight increase in sensitizing action with ammonia, but hardly enough to justify its use.

\section{ERYTHROSIN}

Erythrosin is stated by Eder ${ }^{16}$ to be the best of the flourescin dyes for sensitizing. Other important representatives of this class are eosin and rose bengal. He also states that plates bathed with erythrosin are superior to plates in which the dye is incorporated in the emulsion. We were not able, however, with the sample of erythrosin used (Kahlbaum) to produce plates which were as sensitive in the yellow-green as the orthochromatic plates made by one American manufacturer. (Fig. I5.)

We found erythrosin to give the best results when used according to the directions given by Eder. ${ }^{17}$ The plate is given a prebath for two minutes in

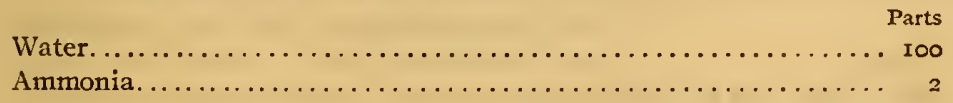

Then it is bathed for two or three minutes in a bath of

Parts

Water........................................ 100

Stock solution (x to rooo).......................... 20

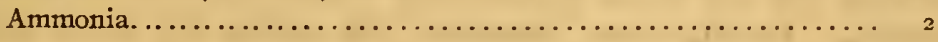


The bathed plates should be washed in water to remove the excess dye from the gelatine. A rinse in alcohol will shorten the time required for drying.

An inspection of the spectrograms of commercial orthochromatic plates indicates that erythrosin or a dye of the erythrosin type is used in their manufacture. The advantage of erythrosin over such dyes as pinaverdol is its limited region of sensitizing action, since the sensitiveness conferred by erythrosin ceases abruptly at $580 \mu$ while the action of the other dyes continues rather farther into the red. This limited region of sensitiveness makes it possible to develop plates made sensitive with erythrosin in the light of an ordinarily good red dark-room lamp. This is a decided advantage to the practical photographer. One of the reasons that panchromatic plates have not been more generally used is that they must be developed in total, or at best in slightly modified darkness, so that the photographer is unable to judge whether or not he is carrying development to the contrast required by the subject and the printing medium to be used.

\section{PINAVERDOL, PINACHROME, ORTHOCHROME T, AND HOMOCOL}

These dyes are very much alike in the regions for which they sensitize. (Figs. I6 and I6a.) Of these four dyes, homocol has its sensitizing action increased most by the use of ammonia. Their greatest application probably lies in their use in conjunction with pinacyanol to supply the slight deficiency of the latter in the green.

\section{HYPERSENSITIZING COMMERCIAL PANCHROMATIC PLATES}

The well-known action of a bath of dilute ammonia in increasing the speed of an ordinary dry plate was applied to commercial panchromatic plates in the process worked out by S. M. Burka ${ }^{18}$ and C. C. Kiess at this Bureau in r9r8. They found that commercial panchromatic plates bathed for four minutes in-

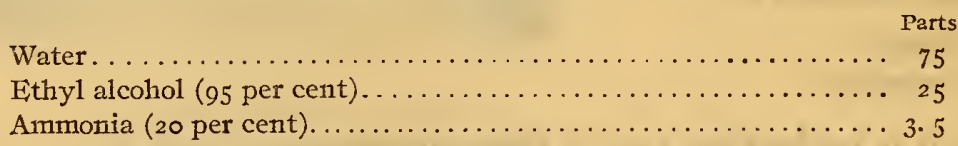

had the total speed increased roo per cent and the red speed increased 200 to 800 per cent. A greater increase in speed was obtained by the use of 3.5 to $100 \mathrm{cc}$ of water, with the omission of the alcohol. In this case, however, the plates dry more slowly

18 Jour. Frank. Inst., 189, p. 25: 1920. 
Scientific Papers of the Bureau of Standards, Vol. 17

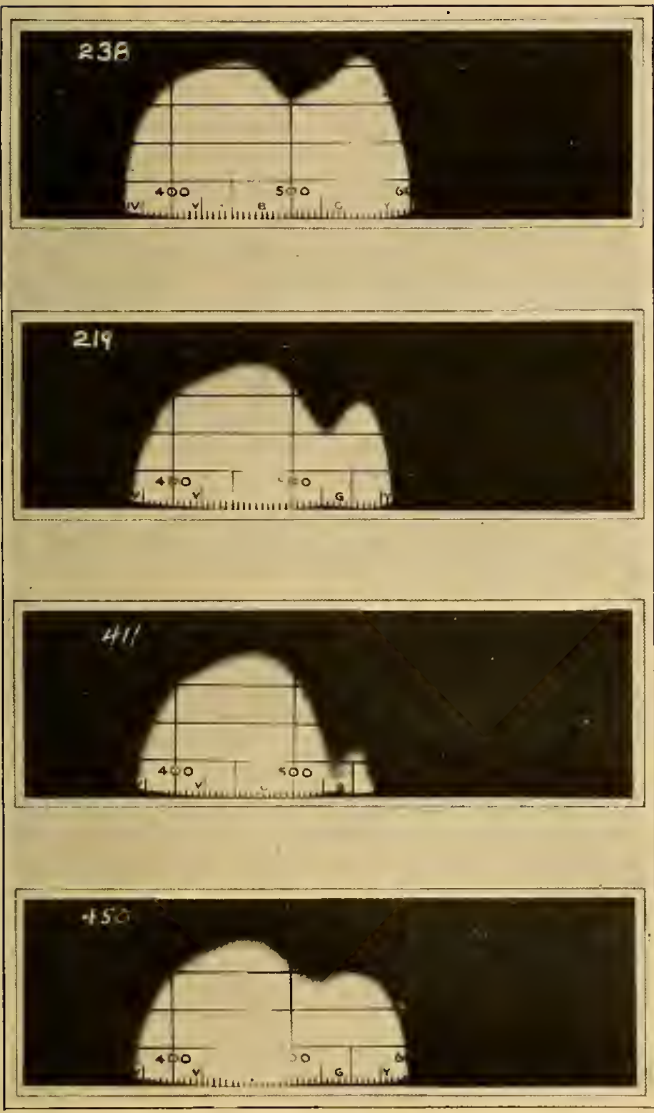

Isochromatic plate

Orthochromatic plate

Orthochromatic film

Plate bathed with erythrosin

FIG. I5.-The comparison of commercial orthochromatic plates with a plate bathed with erythrosin shows that the latter is better than most commercial orthochromatic plates, but not as good as certain oithers 


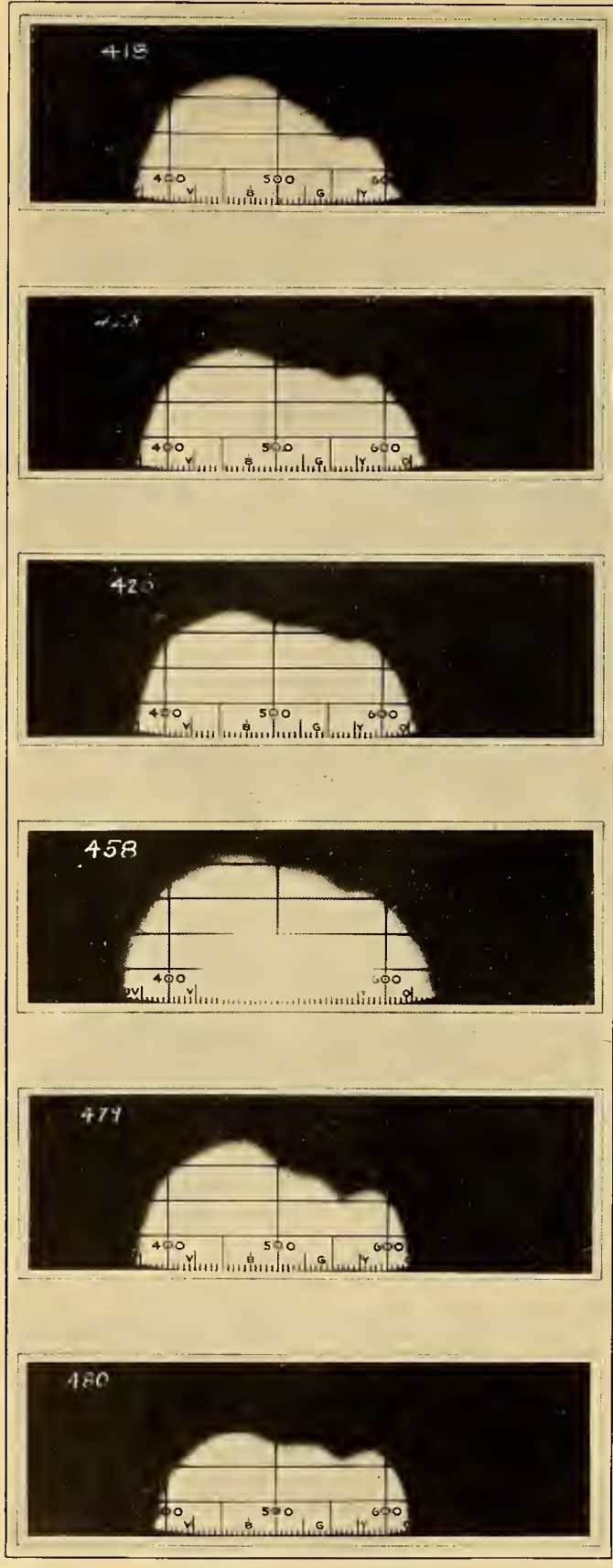

FIG. I6.-Orthochromatic sensitizers used in water bath with and without ammonia

The grcatest effect due to ammonia is found with homocol; the other two dyes show a little extension toward the red. A comparison of these sensitizers with erythrosin shows why they are not used for orthochromatic plates. It would be difficult to devise a dark-room light which would not give considerable fog with them, while with erythrosin the sensitiveness does nct extend beyond 600
Homocol without ammonia

Homocol with ammonia

Pinaverdol without ammonia

Pinaverdol with ammonia

Orthochrome $T$ without arnmonia

Orthochrome $T$ with ammania 
Sclentific Papers of the Bureau of Standards, Vol. 17

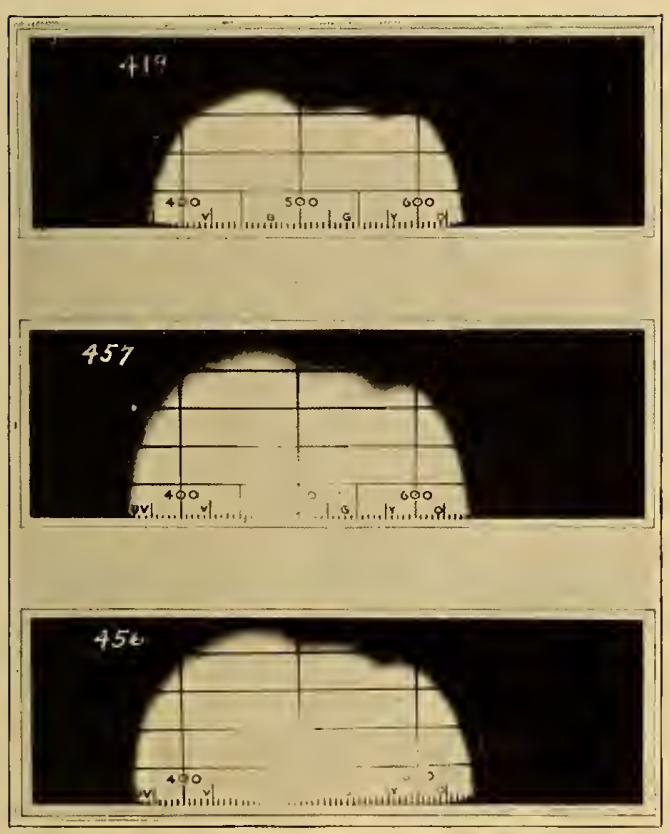

Pinachrome, water only

Pinachrome, water, and ammonia

Pinachrome, water, alcohol, and ammonia

Fig. 10a.-Pinachrome 
and develop more fog than when alcohol is included in the hypersensitizing bath. A rinse in alcohol after the ammonia bath will help in drying the plates more rapidly. In an early investigation of the sensitizing properties of cyanin Schumann found that bathing in a dilute solution of ammonia imparted great speed and sensitivity to a plate with cyanin incorporated in the emulsion. (See Photographische Rundschau, 3, pp. I 43, I 75; 1889.)

In Fig. 17 are shown the results of a keeping test made on Wratten Panchromatic Process, D. C. The plates were hypersensitized without the use of alcohol. The plates gave evidence of a slight increase in speed for a few days after hypersensitizing,

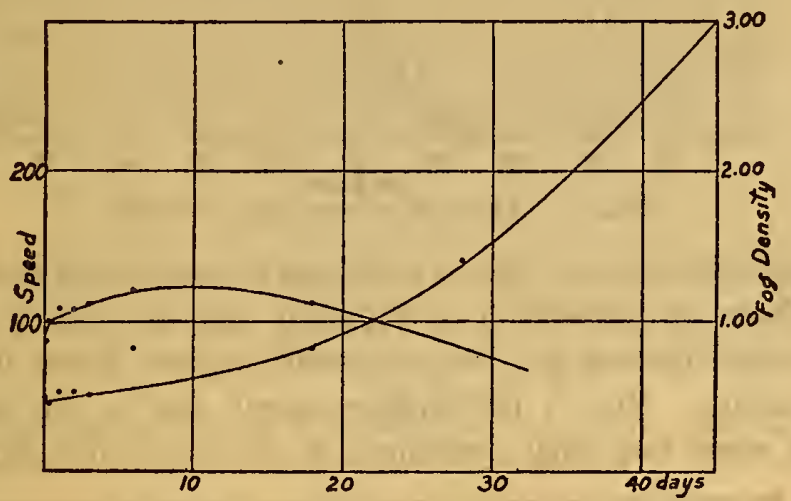

FIG. 17--Variation of speed and fog with time of keeping hypersensitized plates

The fog has increased from a density of 0.45 to 3.00 in 45 days (a density of 3.00 transmits $\mathrm{r} / \mathrm{r} 000$ of the incident light).

NoTE. - The fog is given for $=\gamma_{2}$; that is, the development was carried until the contrast was twice that in the subject.

while the fog kept increasing from the time the plates were hypersensitized.

A comparison of the increase in speed brought about by washing and by hypersensitizing commercial panchromatic plates shows that a part of the hypersensitizing action is due to the removal of certain soluble restraining substances by washing. In Table 2 are given the results of washing and hypersensitizing three typical panchromatic plates.

\section{INCREASE IN SPEED BY WASHING BEFORE EXPOSURE}

The improvement in sensitizing by washing the ordinary plate before bathing in pinacyanol-water solution suggested that there might be a corresponding improvement in the speed of commercial panchromatic plates. Accordingly a few typical panchromatic plates were washed for five minutes in running tap water and 

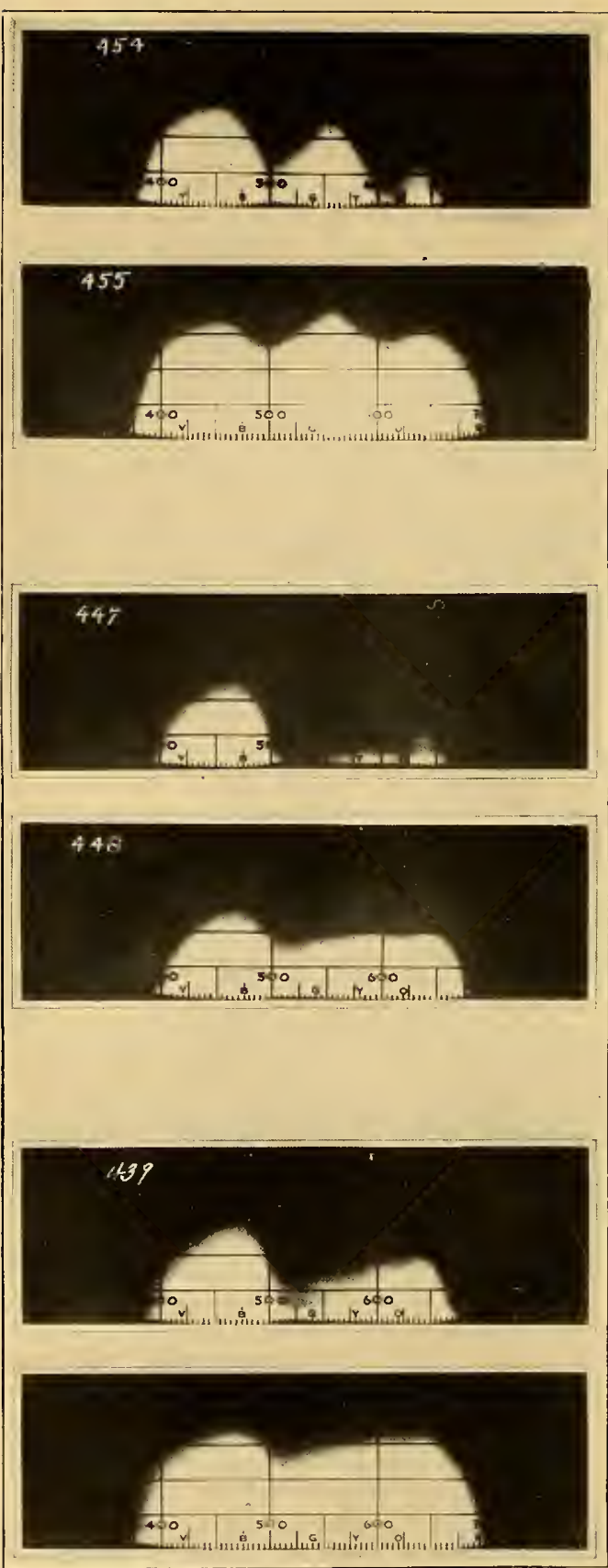

I'IG. I8.- Spectrograms showing increased sensitivity obtained by washing panchromaitc plaies

The plates were washed in water for five minutes, dricd in a cabinct, and exposed. Each was developed simu1tanconsly with an unwashed plate of the same lind $\mathrm{cx}$ posed for the same length of time. The spectrumprocess plate was soaked in distilled water, while the other plates were washed in running tap water. It would appcar that the increase in sensitiveness is clue to the rcmoval of restraining substances from the plate and not to the sencitizing action of any substance iz the water
Spectrum process, unwashed; 20 milnute exposurs

Spectrum process, washed; 20 minute exposure

Panchromatlc, d.c. unwashed

Panchromatic, d.c. washed

Pan-ortho, unwashed

Pan-ortho, washed 
dried, following which the total speed to white light and the filter factors were measured. As seen from Table 2, all of the panchromatic plates showed decided improvement on being washed.

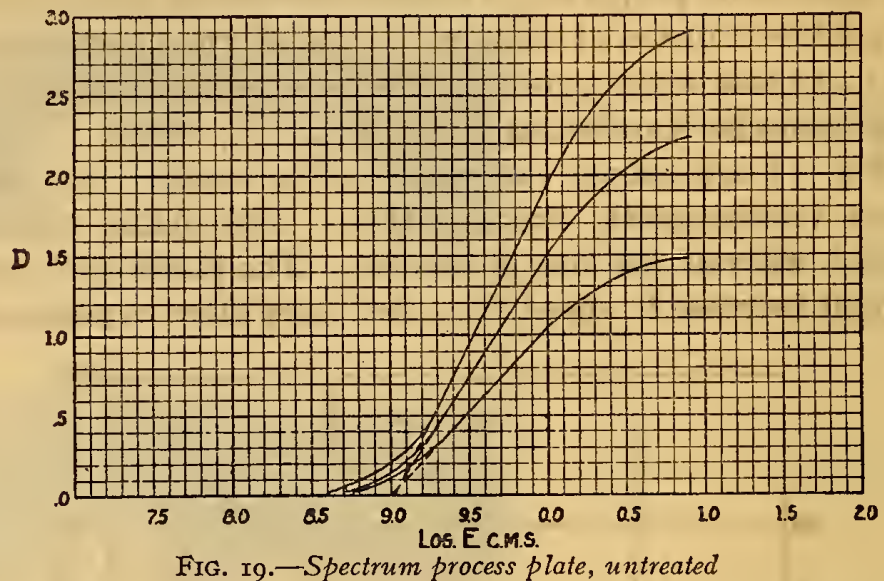

That the effect is not due to a change in the rate of development is clear from an inspection of Figs. I9 and 20, which show the characteristic curves for the spectrum process plate before and after washing. Nor is the improvement due to the sensitizing action of some impurity contained in the tap water, since plates

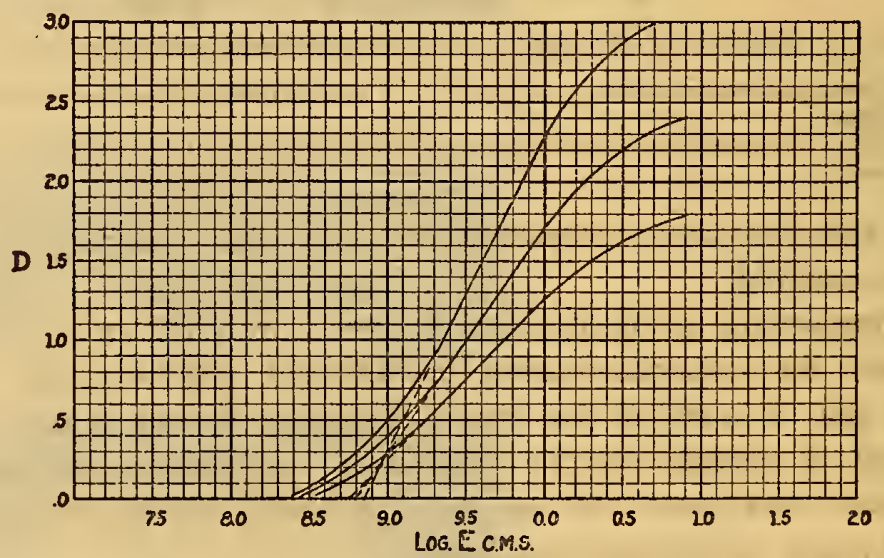

FIG. 20.-Specirum process plate, washa' in tap water for five minutes

washed in a few changes of distilled water showed the effect as well if not better than with tap water. That the action is not due to a variation of the water content of the plate may be concluded from the fact that the plates before exposure in the sensitometer were backed and allowed to dry for about an hour. This time is sufficient for the unwashed plate taken from the box to come to the same state of humidity as the plate which had been washed. 
This increase of sensitiveness may then be concluded to result from the removal of certain restraining substances from the emulsion. From our experience with pinacyanol, chrome alum, and potassium bromide suggest themselves as the restraining substances.

The comparison of the spectrograms of unwashed and washed panchromatic plates (Fig. I8) shows that the greater part of the increase in speed to white light comes in the region of sensitiveness

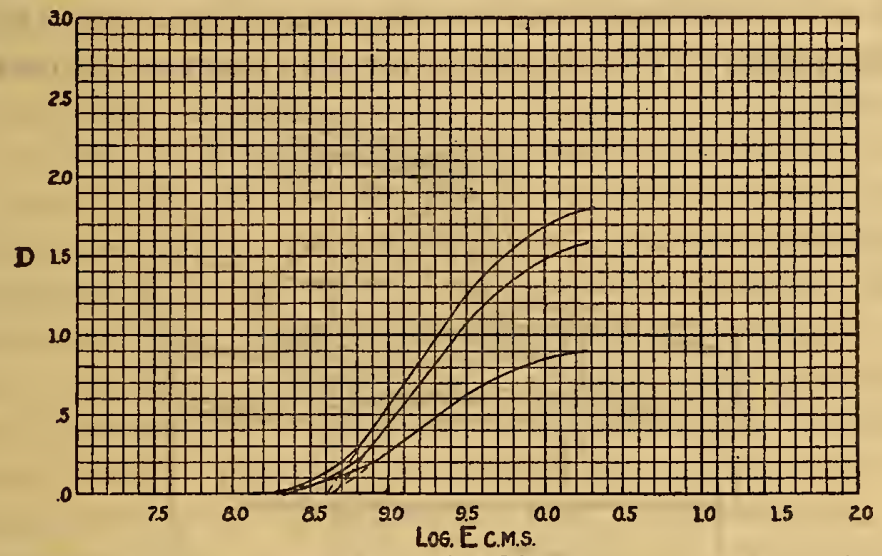

FIG. 21.-Spectrum process plate, hypersensitized in water Ioo cc, ammonia (20 per cent) $3.5 \mathrm{cc}$, and bathed four minutes

which is due to the dyes. This is borne out by the speed measurements with the filters.

TABLE 2

\begin{tabular}{|c|c|c|c|c|c|c|c|}
\hline \multirow{2}{*}{ Plate } & \multirow{2}{*}{ Treatment } & \multirow{2}{*}{$\begin{array}{l}\text { Total } \\
\text { speed }\end{array}$} & \multicolumn{4}{|c|}{ Speed with filters } & \multirow{2}{*}{$\begin{array}{c}\text { Fog den- } \\
\text { sity for } \\
\gamma=1\end{array}$} \\
\hline & & & $\mathrm{A}$ & B & c & G & \\
\hline \multirow[t]{3}{*}{ Spectrum process a.... } & None........... & 96 & 5 & 8 & 8 & 16 & 0.40 \\
\hline & Washed............ & 155 & 13 & 19 & 11 & 40 & .40 \\
\hline & Hypersensitized.. & 235 & 22 & 34 & 16 & 75 & 1.30 \\
\hline \multirow{3}{*}{$\begin{array}{c}\text { Wratten } \\
\text { D. C. }\end{array}$} & None..................... & 180 & 16 & 15 & 17 & 36 & .20 \\
\hline & Washed.................... & 240 & 32 & 26 & 19 & 80 & .40 \\
\hline & Hypersensitized........ & 380 & 59 & 50 & 44 & 140 & .50 \\
\hline \multirow[t]{3}{*}{ Pan-ortino. } & None................... & 91 & 9 & 7 & 8 & 16 & .30 \\
\hline & Washed................ & 145 & 25 & 15 & 10 & 45 & .40 \\
\hline & Hypersensitized...... & 275 & 49 & 32 & 19 & 106 & 1.30 \\
\hline
\end{tabular}

a The spectrum process and the pan-ortho plates were over a year old, while the Wratten plate had just been received from the factory.

The table shows that while washing does not bring the increase in speed that hypersensitizing does it does not cause the production of as much fog. The characteristic curves (Figs. 19, 20, 2r) 
show that washing improves the scale or rendering power of the plate, while hypersensitizing does the reverse.

Manufacturers are urged to investigate the increase of sensitiveness found by washing in water, as it may be of considerable advantage to so treat their panchromatic plates before placing them on the market.

\section{THE TECHNIQUE OF PLATE BATHING}

More or less difficulty will be experienced by anyone learning to bathe plates. It is advisable for the beginner to carry out

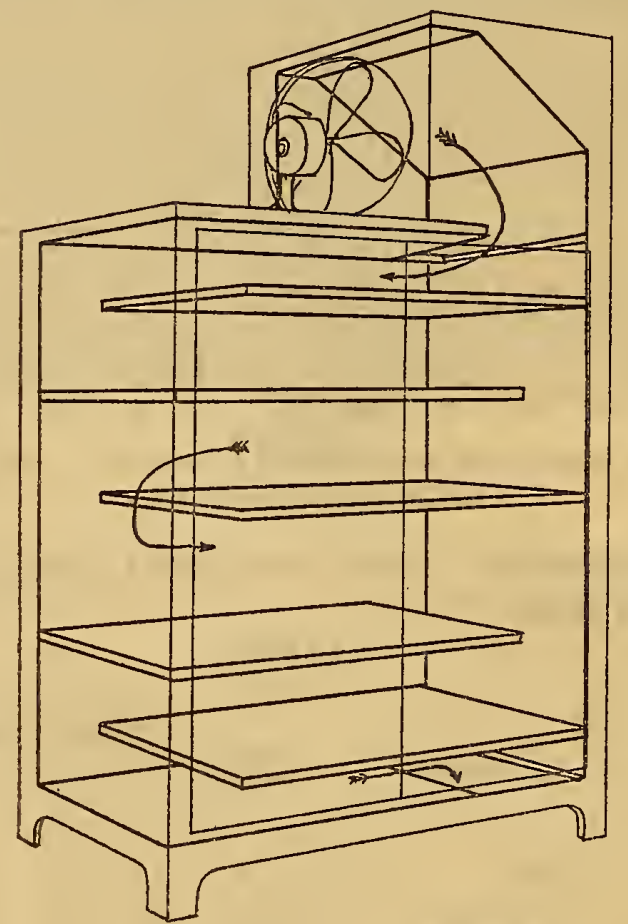

FIG. 22.-Drying cabinet

The outside of the cabinet is shown in light line and the inside in heavy line.

the process in full light. This will enable him to follow the process and to discover the effect of the different baths on the plate; whether or not the bathing is sufficiently long for the dye to get through the emulsion, and whether or not the rinse removes the dye well enough from the gelatine. It will tell him how much he has to rock the trays containing the baths and will give him some idea of how long it takes the plate to dry after bathing. 
An essential of successful sensitizing is absolute cleanliness. Dust or particles of old dye baths remaining in the trays will cause spots and streaks. Glass trays are to be preferred to enameled trays because of the smoother surface and the less likelihood of contamination.

Foggy plates may be due to a number of causes-the choice of the wrong brand of plates for sensitizing, stray light during bathing and drying, the use of baths at too high a temperature, slow drying, or too much ammonia in the dye bath.

Slow drying sometimes gives rise to areas of varying sensitiveness on the plate, depending upon which part of the plate dries first. The rate of drying may be increased by the use of the alcohol rinse and by the use of a properly designed drying cabinet.

The drying cabinet shown in Fig. 22 has several advantages. The air driven in at the top by an electric fan passes through efficient light traps, both at the top and the bottom. Two doors form the front of the box and opening them gives access to all of the shelves, of which there are four, to hold drying racks filled with plates. The upright form of the cabinet gives it a very large capacity for the amount of floor space required. The long distance the air has to travel gives uniform distribution of the air current to all parts of the box in which plates may be placed.

\section{PROCEDURE AND FORMULAS}

I. Stock solutions.-Stock solutions are usually made with pure ethyl alcohol ( 90 per cent or stronger). Some dyes are water soluble (erythrosin), wlile others dissolve in methyl alcohol. The usual strength is I $\mathrm{g}$ of dye to $1000 \mathrm{cc}$ of alcohol (or in the same proportion). Some dyes are less soluble than this and require from 2000 to $10000 \mathrm{cc}$ of alcohol to dissolve $\mathrm{I} g$ of dye. It is advisable to put the dye in the solvent instead of pouring the solvent on the dye, as some of the dye may stick to the bottom of the bottle and require considerable more shaking to get into solution. If the dye does not dissolve in cold alcohol, the bottle containing the dye may be placed in a water bath and heated until the alcohol boils. If it does not go into solution after boiling 30 minutes, more alcohol should be tried.

2. Water solutions. - The bath is composed of

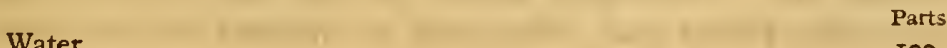

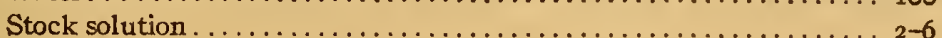


Pinacyanol.-Wash the plate five minutes before bathing. Bathe two minutes. Rinse in alcohol until the film feels hard (three to five minutes).

Pinaverdol, Pinachrome, Orthochrome T.-Prewash optional. If prewash is omitted, bathe for four minutes.

Homocol.-Prewash optional, bathe four minutes if omitted. For increased sensitizing action, add $2 \mathrm{cc}$ of ammonia.

3. Water, alcohol, and ammonia solutions. To be used when water dye bath flocculates on adding ammonia.

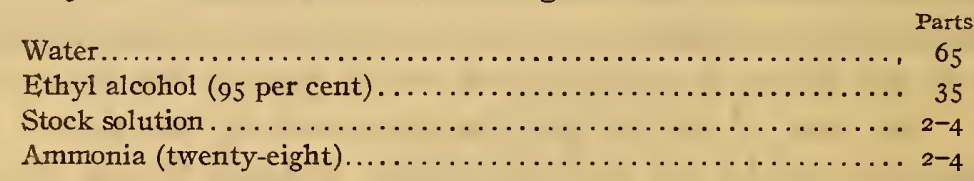

In mixing this bath allow the alcohol and water to cool after mixing, add the stock solution, stir well, and then add the ammonia. Some baths work better if the minimum amount of ammonia is added before bathing the first plate, and more ammonia added as the bath loses its ammonia.

The plates are put directly in this bath, care being taken that the bath covers the entire plate. Bathe for four or five minutes and rinse in alcohol until the film is hard. Temperature should be $18^{\circ} \mathrm{C}$. or less.

Pinacyanol.-As directed.

Dicyanin.--Requires full amount of ammonia.

\section{SUMMARY}

An investigation of the methods of sensitizing ordinary plates by bathing shows that certain precautions are necessary with some of the modern photosensitizing dyes and not with others.

Dicyanin gives a much greater sensitiveness and extends farther into the infra-red when used in a dilute alcoholic solution with ammonia than when used with water alone.

Pinacyanol gives good results in dilute alcoholic solution with ammonia, but plates bathed in water and stock solution are almost as sensitive and keep much better, provided the plates are thoroughly washed before sensitizing, as the soluble salts contained in the emulsion prevent the sensitizing action by flocculating the dye.

Washing has a favorable action on the color sensitiveness of panchromatic plates and, while not so marked as the action of 
dilute ammonia, it gives no increase in fog, which always follows the use of ammonia.

The modern orthochromatic sensitizers, pinachrome, pinaverdol, homocol, and orthochrome $T$, are much less sensitive to electrolytes than are pinacyanol and dicyanin. Ammonia may be added to a staining bath of water and stock solution only, but does not increase the sensitizing action appreciably except in the case of homocol.

Films are best sensitized in a bath containing water, alcohol, ammonia, and stock solution.

Certain brands of orthochromatic plates were found to be superior to plates bathed with erythrosin (Kahlbaum's), but no panchromatic plate compared favorably with pinacyanol bathed plates, except one experimental emulsion furnished by an American manufacturer.

WASHINGTON, July I2, I92 I. 